\title{
Reflexiones docentes acerca de las concepciones sobre la evaluación del aprendizaje y su influencia en las prácticas evaluativas
}

\author{
Teacher reflections about conceptions about the evaluation of learning and its \\ influence on evaluative practices
}

\section{Reflexões de professores sobre concepções sobre a avaliação da aprendizagem e sua influência nas práticas avaliativas}

\author{
Elvira Patricia Flórez Nisperuza ${ }^{1}$ \\ Julio César Páez García² \\ Carlos Mario Fernández ${ }^{3}$ \\ Jhan Freddy Salgado ${ }^{4}$
}

Recibido: junio de 2018

Aceptado: diciembre de 2018

Para citar este artículo: Flórez Nisperuza, E.P., Páez, J., Fernández, C.M., y Salgado, J.F. (2019). Reflexiones docentes acerca de las concepciones sobre la evaluación del aprendizaje y su influencia en las prácticas evaluativas. Revista Científica, 34(1), 63-72. Doi: https://doi.org/10.14483/23448350.13553

\section{Resumen}

Este artículo se enfoca en la evaluación del aprendizaje y su influencia en las prácticas evaluativas a partir del reconocimiento de las concepciones que tienen los estudiantes y docentes implicados en el acto educativo. Esto, fundamentado en investigaciones y opiniones de diferentes autores acerca del tema tratado. Se detalla en los hallazgos investigativos que ponen de relieve las reflexiones construidas por un equipo de docentes y maestros en formación del departamento de Ciencias Naturales y Educación Ambiental de la Universidad de Córdoba (Colombia), en el marco del proyecto de investigación "Caracterización de las prácticas evaluativas en las instituciones educativas del departamento de Córdoba". Se realizó un proceso de búsqueda e identificación de información documental en bases de datos, categorizadas y analizadas a través de instrumentos de rúbrica y glosa partiendo de un método heurístico (que conllevó a la preparación, selección y búsqueda). Lo anterior permitió obtener criterios de caracterización de cada investigación. En una posterior fase hermenéutica, se realizaron reflexiones documentadas y contrastadas para constituir las conclusiones. Finalmente, se establece que las concepciones de los docentes y estudiantes tienen relevancia recíproca. Los docentes son los encargados de asesorar el proceso evaluativo y determinan si se ha alcanzado un aprendizaje significativo; de otra parte, los estudiantes, desde la concepción

\footnotetext{
Universidad de Córdoba. Montería, Colombia. Contacto: epatriciaflorez@correo.unicordoba.edu.co

Universidad de Córdoba. Montería, Colombia. Contacto: juliopaez@correo.unicordoba.edu.co

Universidad de Córdoba. Montería, Colombia. Contacto: carfers07@gmail.com

Universidad de Córdoba. Montería, Colombia. Contacto: jhanfreddysalgado@gmail.com
} 
de conocer, actúan como agentes pasivos o activos en el proceso evaluativo. Además, la evaluación es tomada como un medio que permite determinar las debilidades y fortalezas que posee tanto el docente como el estudiante. Palabras clave: concepciones, evaluación, proceso, enseñanza, aprendizaje, prácticas.

\section{Abstract}

This article deals with the evaluation of learning and its influence on evaluative practices, based on the recognition of the conceptions of students and teachers involved in the educational act, based on research and opinions of different authors on the subject. It is detailed in the research findings that highlight the reflections built by a team of teachers and teachers in training from the Department of Natural Sciences and Environmental Education of the University of Córdoba -Colombia, within the framework of the research project "characterization of evaluation practices in the Educational Institutions of the Department of Córdoba". A search and identification process of documentary information is carried out in databases, categorized and analyzed through rubric and glossary instruments based on a heuristic method that led to the preparation, selection and search, allowing obtaining characterization criteria for each investigation. And arriving at a hermeneutic phase, in which documented and contrasted reflections were made to reach conclusions. Finally, it is established that the conceptions of teachers and students have reciprocal relevance, where teachers are in charge of assessing the evaluation process and determine if significant learning has been achieved; and students, from the conception of knowledge, act as passive or active agents in the evaluation process. In addition, the evaluation is taken more than as an instrument, as a means to determine the weaknesses and strengths that both the teacher and the student have.

Keywords: conceptions, evaluation, process, teaching, learning, practices.

\section{Resumo}

Este artigo trata da avaliação da aprendizagem e sua influência nas práticas avaliativas, a partir do reconhecimento das concepções de alunos e professores envolvidos no ato educativo, a partir de pesquisas e opiniões de diferentes autores sobre o tema. Está detalhado nos achados da pesquisa que destacam as reflexões construídas por uma equipe de profesores e professores em formação do Departamento de Ciências Naturais e Educação Ambiental da Universidade de Córdoba-Colômbia, no âmbito do projeto de pesquisa "caracterização das práticas de avaliação nas instituições de ensino do departamento de Córdoba". um processo de busca de informação e documento de identificação é realizada em bancos de dados, categorizados e analisados por meio de secção de instrumentos e glosses com base num método heurístico que conduziu à preparação, selecção e pesquisa, permitindo a obtenção de critérios para a caracterização de cada investigação e chegando a uma fase hermenêutica, na qual reflexões documentadas e contrastadas foram feitas para chegar a conclusões. Por fim, estabelece-se que as concepções de professores e alunos têm uma relevância recíproca, em que os professores são responsáveis por avaliar o processo de avaliação e determinar se a aprendizagem significativa foi alcançada; e os estudantes, a partir da concepção de saber, atuam como agentes passivos ou ativos no processo de avaliação. Além disso, a avaliação é tomada mais do que como um instrumento, como um meio para determinar as fraquezas e forças que tanto o professor quanto o aluno têm.

Palavras-chaves: concepções, avaliação, processo, ensino, aprendizagem, práticas.

\section{Introducción}

Reflexionar sobre la práctica docente es un Ilamado profesional permanente por parte de los actores que intervienen en el campo de la educación. De manera especial, docentes y estudiantes en quienes se sostiene la responsabilidad formativa de impacto en la sociedad, integrada por un entramado de categorías conceptuales que le dan sentido a la educación en sus distintos niveles y áreas del saber. El currículo, la didáctica y la evaluación se constituyen en aspectos determinantes que estructuran el discurso pedagógico y legitiman en gran 
medida la institucionalidad; a la vez que definen la teoría y la práctica educativa. Sobre esta última resulta imprescindible desarrollar procesos de investigación que enriquezcan el acto educativo a partir del reconocimiento de los propios actores implicados.

El interés en la evaluación como campo de reflexión del ejercicio docente del presente artículo tiene profundas vinculaciones con el acto de enseñar y de aprender, incluso con el de ser profesionales. Así lo reportan teóricos como Santos-Guerra (1998), en quien se defiende la necesidad de renovados procesos de reflexión y que hacen eco en las siguientes líneas que describen las ganancias conceptuales logradas al interior de la primera fase del proyecto de investigación titulado "Caracterización de las prácticas evaluativas de las Instituciones Educativas del departamento de Córdoba como campo de formación en el estudiante de la Licenciatura de Ciencias Naturales y Educación Ambiental de la Universidad de Córdoba", consistentes en develar las investigaciones rastreadas relacionadas con las concepciones sobre la evaluación del aprendizaje, que muestran el estado actual en este campo y se convierten en el punto de partida clave para el proceso de reconocimiento y de descripción del contexto evaluativo en el actuar docente.

Los alcances de esta primera fase se sustentan, además, por el interés mostrado en los maestros en formación participantes en esta experiencia investigativa. En estos subsiste una permanente preocupación por adentrarse en las realidades que acontecen en las instituciones educativas Lo anterior, desde una perspectiva que va más allá de lo que han observado en su rol de practicantes; por lo que se logró evidenciar la permanencia de prácticas evaluativas reduccionistas. Conforme a la esperanza pedagógica de enriquecer la premisa teórica que reposa de manera concreta sobre la evaluación del aprendizaje como categoría que pone al descubierto el docente que somos. Ello coincide con Sacristán (1995) cuando afirma que estudiar la evaluación trae consigo el reconocimiento de la pedagogía en su complejidad, de sus relaciones conceptuales configurativas del trabajo docente y, en particular, de toda la pedagogía que se practica.

El rastreo de las investigaciones sobre las concepciones de evaluación del aprendizaje que se han hecho a nivel nacional e internacional viene a ser el primer avance en la caracterización de las prácticas evaluativas. Luego, se ejecutaron dos procesos de reflexión inherentes en toda investigación: el heurístico, caracterizado por la indagación, la búsqueda, la selección y la organización de investigaciones afines a la problemática expuesta; y el hermenéutico, caracterizado por la interpretación y el análisis, a modo de hilar un discurso de cara a lo planteado.

En este sentido, las pesquisas construidas dieron como resultado tres categorías que sustentan el estado actual de la evaluación del aprendizaje y que son la flecha que apunta a un norte específico en la intención de caracterizar las prácticas evaluativas, a partir de lo que existe en teoría, para entregar nuevos sentidos a lo cotidiano y, en especial, a los renovados y esperados modos de pensar y hacer de la evaluación. Estas son:

1. Reflexiones acerca de las concepciones de docentes y estudiantes, como punto de partida para asumir la evaluación dentro del acto educativo.

2. Reflexiones acerca de las concepciones de los docentes sobre la evaluación del aprendizaje, como punto de partida para integrar su discurso en el aula.

3. Reflexiones acerca de las concepciones de los docentes sobre la evaluación del aprendizaje y sus implicaciones en las prácticas evaluativas en el contexto de las ciencias naturales, como punto de partida para el aprendizaje con sentido.

A continuación, se explican cada una de estas categorías. 


\section{Reflexiones acerca de las concepciones de docentes y estudiantes, como punto de partida para asumir la evaluación dentro del acto educativo}

Abordar el panorama en el que se circunscribe el docente en relación con la evaluación implica una mirada global acerca de las concepciones que se han dado al respecto. Para Villota y Rosero "la evaluación debe entenderse como un soporte permanente al desarrollo de la persona y un continuo quitar obstáculos para que el individuo encuentre su plena realización" (1999). Desde esta perspectiva, la evaluación implica un proceso que señala unas directrices para pensar en la formación del estudiante, no solo desde el campo intelectual, sino también desde las diferentes dimensiones que lo caracterizan como ser humano.

Davis, señala que "la evaluación es un procedimiento sistemático para recopilar información acerca de los estudiantes" (citado por López, 2014). Esta información se convierte en el insumo que tiene el docente para dar o emitir un juicio de valor acerca de los estudiantes, dado que en ella se ha de evidenciar el desarrollo de las habilidades a las que ha llegado este en su proceso de formación integral.

Al respecto, Gómez y Gamero señalan que "a pesar de la existencia de unos lineamientos generales que orientan la práctica evaluativa, los docentes influenciados por sus creencias le dan matices muy diferentes al proceso evaluativo" (2016, p. 12). Esto ocurre, quizás, por la interpretación que hacen de la misma. Si bien la evaluación es un proceso permanente, en el tránsito de este se debe advertir cuáles son los aspectos que se han de mejorar y no postergar este proceso hasta el final con la intención única de recoger información con la que el maestro determina si se aprueba o no dicho proceso. Indiscutiblemente, estas creencias marcan un derrotero y hacen un gran aporte para conocer el desarrollo de las habilidades de los estudiantes en su proceso de enseñanza-aprendizaje.
Partiendo de lo expuesto, es evidente que el docente constituye un punto de quiebre al momento de crear la coherencia entre lo que se dice, se hace y lo que se debe hacer. Debido a esta dificultad la comunidad educativa en general, y sobre todo los docentes, deberán reflexionar y buscar estrategias conformes al proceso constante para evaluar el desempeño del estudiante. Se evalúa con el propósito de contribuir al desarrollo y formación integral del maestro, con el fin de favorecer el aseguramiento de sus aprendizajes y así mejorar la calidad de la educación.

La importancia de los procesos evaluativos en las técnicas de enseñanza-aprendizaje en las aulas de clases. Además, presenta un análisis sobre la realidad teórico-práctica que se tiene en las instituciones educativas a través de un estudio de casos muy complejo en el que tiene en cuenta las concepciones de autores y expertos en el tema y considera el contexto y realidad que se viven en esta década en relación con los procesos educativos de las instituciones. También tiene en cuenta diversas estrategias y metodologías que se pueden desarrollar al momento de llevar a cabo cualquier práctica evaluativa en los procesos de enseñanza-aprendizaje.

Por otro lado, las concepciones de los estudiantes juegan un papel de gran importancia en lo que concierne al aprendizaje, pues de estas se derivan algunos aspectos fundamentales para su proceso de formación. Señoriño, Patat, Vilanova y García afirman que:

Puede considerarse que uno de los factores decisivos que hacen que un estudiante participe de manera constructiva y crítica en el aprendizaje, es la propia concepción que tiene acerca de la adquisición del conocimiento. Esta concepción sobre lo que es conocer, puede inducirlo a verse a sí mismo como un mero receptor y espectador pasivo o, por el contrario, como constructor y participante activo de su propio aprendizaje. (2014, p. 131)

Ahora bien, pensar en la evaluación del aprendizaje implica tener en cuenta las concepciones 
que cada uno de los estudiantes posee, entendiendo que cada individuo tiene su propia forma de adquirir el conocimiento. Así lo evidenciaron los autores antes mencionados, al determinar cómo actúan los estudiantes en diferentes espacios que se pueden presentar dentro del aula, lo cual brinda al docente varias maneras de poder llegar y enseñar a los estudiantes de forma adecuada, logrando en ellos el interés por aprender para asegurar así un aprendizaje significativo.

A partir de lo anterior, se puede inferir que las concepciones de los docentes y estudiantes presentan una doble relevancia práctica. De un mismo proceso se desprenden dos concepciones con una reciprocidad expresada, por una parte, por los docentes, quienes son los responsables de asesorar el proceso evaluativo y determinar si se ha alcanzado un verdadero aprendizaje significativo; $y$, por la otra, los estudiantes quienes, a partir de la concepción de conocer, actúan como agentes pasivos o activos en el proceso evaluativo.

\section{Reflexiones acerca de las concepciones de los docentes sobre la evaluación del apre- ndizaje, como punto de partida para inte- grar su discurso en el aula}

La concepción de los docentes sobre la evaluación de los aprendizajes va dirigida al proceso permanente de la perfección, buscando la excelencia de todos los componentes en formación, orientándose hacia el desarrollo de los procesos de enseñanza-aprendizaje. La investigación realizada en la Universidad de Caldas por Ruíz, Dussán y Ruíz concluyó que "los estudiantes cursan asignaturas de formación general en la universidad; en ellas, se tocan aspectos conceptuales básicos sobre la enseñanza, el aprendizaje y la evaluación sin hacer distinciones entre las disciplinas para las cuales se forman como docentes" (2014, p. 158). Lo que permite identificar dificultades en el proceso de enseñanza-aprendizaje, en el cual se debe intervenir en los diferentes programas de formación con el fin de planificar estrategias para el mejoramiento continuo.

Partiendo de lo anterior, se estudian las concepciones que tiene el maestro en formación con respecto a la evaluación del aprendizaje, estableciendo las vinculaciones con la práctica educativa. Esto permite que se analice el contexto con el propósito de formular lo que todo estudiante debe saber y saber hacer, con el fin de cambiar la perspectiva que se tiene en el proceso educativo. Esto resulta de alto interés para que el docente tome una posición positiva hacia el aprendizaje, la formación, la definición de principios profesionales y la innovación para acceder a una educación de calidad. Cabe señalar los procesos evaluativos que existen, así como los roles que desempeñan los miembros de las comunidades educativas en la formulación y realización de estos.

La evaluación es una actividad esencialmente subjetiva y valorativa, la cual es un reflejo de las concepciones de los profesores, de las creencias y conocimientos especializados y son una base relevante para la práctica profesional de los docentes, operando como el fundamento central que guía sus prácticas (Vergara, 2011). La evaluación es un instrumento muy útil y valorativo porque ayuda al docente a detectar fortalezas y debilidades en los estudiantes con el propósito de mejorarlo; es por ello que es de gran importancia que el docente en el aula de clase utilice distintas actividades y estrategias evaluativas al servicio de las múltiples operaciones intelectuales.

Se hace menester que el docente, al momento de realizar el ejercicio evaluativo, se enfoque desde la perspectiva del estudiante y armonice la relación estudiante-evaluación-profesor, tal y como lo señalan Murillo e Hidalgo: "en la medida que conozcamos y comprendamos mejor las concepciones que tienen los estudiantes sobre la evaluación, estaremos en condiciones de desarrollar mejores evaluaciones, aquellas que se ajusten más a sus necesidades y expectativas, y con ello, mejorar su aprendizaje" (2016, p. 108). Es por ello que el docente debe conocer las concepciones que tiene el 
estudiante sobre la evaluación, para así poder desarrollar en ellos un proceso de enseñanza-aprendizaje que se ajuste a las necesidades (debido que son procesos que deben darse atendiendo a las particularidades e individualidades de todo ser humano) y que sirvan, por tanto, para potenciar siempre el desarrollo de las habilidades y las competencias de los maestros en formación. La cuestión radica en cuánto conoce el docente acerca de sus estudiantes, del nivel de aprehensión del conocimiento que tiene y la puesta en función de este en la sociedad para llevar a cabo verdaderos procesos evaluativos que estén en concordancia con la formación que se propende en el docente que ha de liderar procesos cognitivos, sociales y de transformación en un futuro próximo.

La evaluación es un instrumento de gran utilidad en la práctica evaluativa del docente. A través de esta se da seguimiento de los aprendizajes que los estudiantes van teniendo y de su desarrollo personal, por lo tanto:

Se debe tener una guía para evaluar y más si ella, está acorde al propósito propio de la evaluación. De esta manera un manual representa una ayuda al docente, debido a que este, reconoce los elementos teóricos sobre evaluación, le permite poner en práctica actividades que incitan a la reflexión y transformación de sus prácticas evaluativas. (Pérez, Hernández, De Rojas y González, 2012).

Resulta evidente que ante la falta de una cultura de la evaluación y el abuso del discurso calificatorio, se logre una valoración de los aprendizajes, no como el resultado de un proceso sino atendiendo a la reflexión y apreciación de dicho proceso.

En consonancia con lo planteado, se puede afirmar que la evaluación es considerada más que un instrumento en un medio que determina las debilidades y fortalezas que posee tanto el docente como el estudiante durante su proceso de aprendizaje, que incita a la reflexión mutua y continua y a la creación de nuevos escenarios y recursos ajustados a la vida socio-cognitiva y emocional del estudiante. Esta reflexión, sin duda alguna, nos lleva a hacer un Ilamado a los establecimientos educativos para que revisen, de manera minuciosa, esos saberes acerca de las disciplinas en las cuales se forma al estudiante, así como también a quienes aspiran a ser docentes para que se interesen más en cómo están siendo formados. Ha de haber un cuestionamiento permanente frente a los procesos de evaluación para así poder emitir juicios valorativos que apunten a una evaluación lo más fidedigna y objetiva, atendiendo al ser humano en su integralidad y promoviendo alternativas de evaluación pertinentes cada vez que estas sean necesarias.

\section{Reflexiones acerca de las concepciones de los docentes sobre la evaluación del apren- dizaje y sus implicaciones en las prácticas evaluativas en el contexto de las Ciencias Naturales, como punto de partida para el aprendizaje con sentido}

Estudiar las concepciones y prácticas evaluativas de los docentes, en general, requiere procesos de observación y de descripción del profesorado en las instituciones educativas. Esto, a partir del diagnóstico de las metodologías empleadas y orientadas a la valoración de los aprendizajes, de las situaciones escolares, del procesamiento de la información y la toma de decisiones en el contexto.

Al respecto, Turpo (2011) señala que el trabajo que realizan los docentes al evaluar el aprendizaje de sus estudiantes y la reflexión de ese proceso implican dar una mirada hacia "las concepciones y prácticas evaluativas; es decir, describir los procesos que los docentes discriminan, valoran, critican, fundamentan, enjuician y optan, entre lo que considera que tiene un valor en sí y aquello que carece de él" (p. 214). Es decir, la enseñanza debe estar enfocada a lo práctico y llevando con ello al estudiante a ser un profesional que ponga en función esos saberes y valores en la sociedad, transformándola de manera positiva y logrando un alto impacto en la misma. 
Por su parte Oñate, Saavedra y Spolmann mencionan que:

en la dimensión de evaluación de aprendizajes científicos, coexisten distintas concepciones [...] una concepción constructivista que las actitudes del estudiantado hacia la ciencia se pueden evaluar durante el desarrollo de las actividades experimentales, sin embargo, los resultados que obtengan al final del proceso, son los que darán cuenta a través de la evaluación sumativa. (2011, p. 89)

La evaluación es vista como un proceso de aprendizaje permanente en el cual el educador debe desarrollar diferentes actividades para que el alumno edifique su propio sistema de aprendizaje.

Los maestros de Ciencias cuentan con un modo específico para evaluar el aprendizaje durante su quehacer docente; esto da a entender que su subjetividad es un poco compleja. Turpo (2013) afirma que: "Su abordaje supone reducir el foco de atención, al proceso evaluativo desarrollado por los docentes, al discriminar, valorar, criticar, decidir, entre lo que piensan que tiene un valor en sí y lo que carece de él" (p. 230). Así, se puede decir que los docentes de Ciencias tienden a ser más exigentes al momento de evaluar que los docentes de otras áreas.

La subjetividad tiene un gran valor en los docentes de Ciencias y por tal razón en la forma de actuar frente a las diferentes situaciones académicas que suelen presentarse, tal es el caso de la evaluación del aprendizaje. La manera de ver las cosas desde este punto se hace de manera particular, pese a los criterios que se han desarrollado en la formación como docente, es decir, la pedagogía y epistemología (que son las bases fundamentales para situar sus procesos de enseñanza) son dejadas de lado, en ciertas ocasiones. Aspecto que genera un ruptura entre lo que debe ser y lo que es.

Si bien el autor no profundiza en cuanto a la forma cómo se podría manejar la subjetividad en los docentes de Ciencias frente a la evaluación del aprendizaje, deja ver a través de los resultados obtenidos durante el proceso que se deben buscar alternativas que permitan mejorar el ambiente escolar. También señala tener en cuenta qué piensan los alumnos frente a esta situación, en tanto que son los que interactúan directamente con los profesores.

Como resultado de todo lo anterior, la evaluación es un componente esencial del proceso de enseñanza-aprendizaje que permite valorar el cumplimiento de los objetivos cuando se concibe y organiza. Constituye un valioso instrumento mediador y formativo en las concepciones y prácticas evaluativas de los docentes dado su alto impacto social. Lucena y Santos afirman que: "partiendo de esa valoración y de las problemáticas evidenciadas en la Universidad Estatal de Piauí, Brasil, nacen investigaciones con el objetivo de renovar el proceso de la evaluación, partiendo del estudio de antecedentes y referentes teóricos acerca de este tópico" (2012, p. 7).

Es muy importante identificar que el aprendizaje se basa principalmente en las metodologías utilizadas por los docentes. Esto permitió a la investigación formar grupos en los cuales estudiantes y profesores de los cursos de Licenciatura en Ciencias Biológicas y Matemática crearan una metodología didáctica basada en la formación del aprendizaje, con el fin de que los futuros profesionales egresen con las competencias necesarias en el momento que tengan que ejercer su profesión.

Es de gran importancia conocer procesos que señalen cómo profesores y estudiantes pueden discutir y crear nuevos modelos de enseñanza con el fin de llegar a verdaderos procesos de aprendizaje, lo cual ha de contribuir a la formación del futuro licenciado que pueda desempeñarse como un líder que apunta a procesos de transformación de la sociedad con la que interactúa.

Kimberly Brenneman (2011) afirman que los maestros observan, escuchan y hacen preguntas para evaluar las ideas y comprensiones infantiles en medio de las actividades cotidianas de las clases, es por ello que señalan: 
[...] las herramientas basadas en el rendimiento, influenciadas por los comportamientos continuos y productos de trabajo de niños bajo circunstancias no estructuradas y semi-estructuradas, proporcionan una evaluación formativa del aprendizaje infantil y pueden ser utilizadas por maestros para diseñar experiencias instructivas nuevas para apoyar y estimular mejor el aprendizaje de la ciencia y en otras áreas. $(2011$, p. 7$)$

El docente debe diseñar talleres educativos de aprendizaje en el aula de clase para detectar, analizar y valorar los procesos de enseñanzas y aprendizaje que tiene el niño. La evaluación de estos se debe realizar a través de la observación continua. Es deber del docente saber cómo va avanzando el niño en su proceso de enseñanza y aprendizaje, cuál es su ritmo de trabajo y cómo va interiorizando los procesos que se desarrollan.

Frente a este proceso se puede afirmar que existen concepciones subjetivas, dogmáticas y constructivas, unas más arraigadas que otras, las cuales hacen percibir a los profesores de Ciencias Naturales, de alguna manera, como más disciplinados y rígidos al momento de valorar y criticar la formación de los estudiantes a su cargo. La figura 1 da cuenta de las tres categorías y autores con las respectivas reflexiones acorde al análisis realizado.

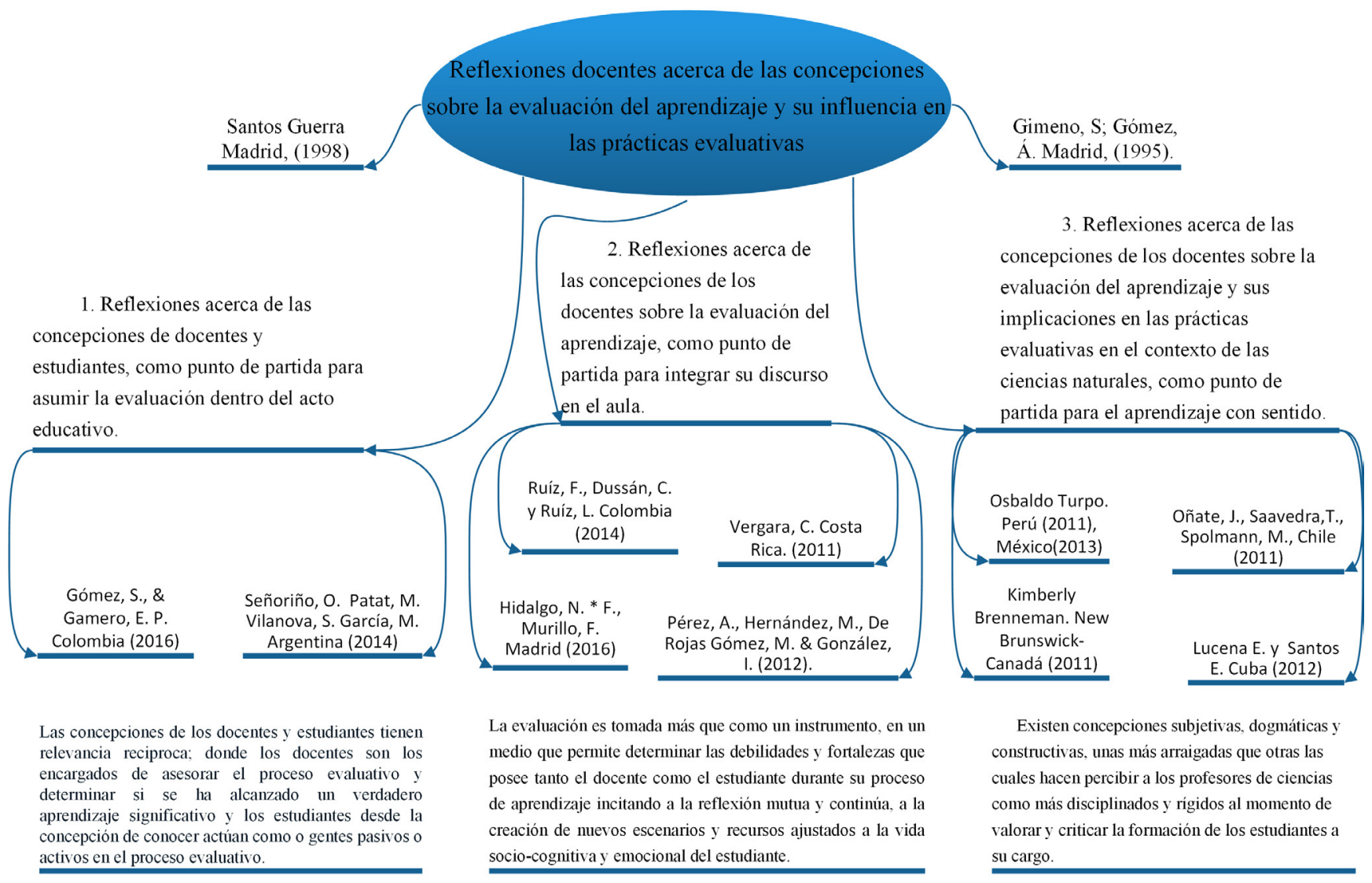

Figura 1. Fuente elaboración propia

Figura 1. Análisis sobre las concepciones docentes de la evaluación del aprendizaje y su influencia en las prácticas evaluativas.

Fuente: elaboración propia. 


\section{Conclusiones}

Ante el análisis y reflexión de las tres categorías identificadas en este documento se puede concluir que:

Las concepciones de los docentes y estudiantes tienen relevancia recíproca. Los docentes son los encargados de asesorar el proceso evaluativo y determinar si este ha sido exitoso y si ha habido un verdadero aprendizaje en los estudiantes; mientras que estos, desde la concepción de conocer, bien pueden actuar como agentes pasivos o activos en el mismo a la hora de ser evaluados.

Se evidencia cómo la evaluación es tomada como, más que un instrumento, un medio que permite determinar las debilidades y fortalezas que posee tanto el docente como el estudiante durante su proceso de aprendizaje. El cual incita a la reflexión mutua y continua, a la creación de nuevos escenarios y recursos ajustados a la vida socio-cognitiva y emocional del estudiante.

Existen concepciones subjetivas, dogmáticas y constructivas, unas más arraigadas que otras, las cuales hacen percibir a los profesores de Ciencias como más disciplinados y rígidos al momento de valorar y criticar la formación de los estudiantes a su cargo.

Por lo anterior, se le hace un Ilamado a los establecimientos educativos y a los aspirantes a convertirse en docentes a meditar sobre las disciplinas para los cuales son formados, de cuestionarse cuando estén frente a la elaboración de instrumentos valorativo. De igual modo, revisar alternativas de evaluación cuando sea necesario y apostarle a una cultura de la evaluación.

\section{Agradecimientos}

A la Universidad de Córdoba por avalar desde el Centro de Investigaciones el proyecto de investigación "Caracterización de las prácticas evaluativas de las instituciones educativas del departamento de Córdoba como campo de formación académico e investigativo en el estudiante de la Licenciatura de Ciencias Naturales y Educación Ambiental de la Universidad de Córdoba". Así mismo, al programa de Licenciatura en Ciencias Naturales y Educación ambiental, al grupo de investigación Gicnea y a los estudiantes y docentes del diplomado Evaluación formativa como estrategia comprensiva. Perspectivas didácticas contemporáneas, cohorte 2018-I, por adentrarse en la organización, búsqueda de información, análisis y reflexión como aportes para identificar las concepciones docentes sobre la evaluación del aprendizaje.

\section{Referencias}

Brenneman, K. (2011). La evaluación del aprendizaje en ciencias y de ambientes de este aprendizaje a nivel preescolar. Early Childhood Research \& Practice, 13(1). Recuperado de http://ecrp.uiuc.edu/v13n1/brenneman-sp. $\underline{\mathrm{html}}$

Gimeno, S. y Gómez, Á. (1995). Comprender y transformar la enseñanza. Madrid: Ediciones Morata.

Gómez, S. y Gamero, E. P. (2016). Concepciones de los docentes sobre la evaluación y su coherencia con los lineamientos institucionales y las prácticas evaluativas. En 3er Simposio Internacional y 4to Coloquio Regional de Investigación Educativa y Pedagógica. 5, 6 y 7 de octubre, Montería (Colombia). Recuperado de http://revistas.unicordoba.edu.co/conferencias/index.php/sue-iep/sue-iep3/paper/ download/77/79

Hidalgo, N. y Murillo, F. (2016). Las concepciones sobre el proceso de evaluación del aprendizaje de los estudiantes. REICE. Revista Iberoamericana sobre Calidad, Eficacia y Cambio en Educación, 15(1), 107-128. Recuperado de http:// www.redalyc.org/pdf/551/55149730007.pdf

López, A. A. (2014). La evaluación como herramienta para el aprendizaje. Conceptos, estrategias y recomendaciones. Editorial Magisterio. Bogotá.

Lucena, S. y Santos, P. (2012). Estrategia didáctica para renovar las concepciones y prácticas de la 
evaluación del aprendizaje en los cursos de licenciatura en ciencias de la universidad estatal de Piauí (tesis de doctorado). Instituto Central de Ciencias Pedagógicas, La Habana. Cuba. Recuperado de http://beduniv.reduniv.edu.cu/ fetch.php?data $=710 \&$ type $=$ pdf \&id $=710 \& d b=1$

Oñate, J., Saavedra, T. y Spolmann, M. (2011). Estudio exploratorio acerca de las concepciones del profesorado deficiencias en formación sobre a evaluación de aprendizajes científicos y resolución de problemas científicos escolares (tesis para optar al grado de licenciado en educación). Pontificia Universidad Católica de Chile. Santiago, Chile. Recuperado de http:// www7.uc.cl/sw educ/educacion/grecia/plano/html/biblioteca/licenciatura.html

Pérez, A. L., Hernández, M. E., De Rojas, M. C. y Gonzáles, I. (2012). Hacia una concepción desarrolladora en la calidad de la evaluación del aprendizaje. Propuesta de Manual. Edumecentro, 4(3), 125-132. Recuperado de http:// scielo.sld.cu/scielo.php?script=sci arttext\&pi$\underline{\mathrm{d}=S 2077-28742012000300014 \& \operatorname{lng}=\mathrm{es} \& \text { tlng=es }}$

Ruíz, F., Dussán, C. y Ruíz, L. (2014). Concepciones de los docentes en formación de la Universidad de Caldas, sobre la evaluación de los aprendizajes. Revista Latinoamericana de Estudios Educativos (Colombia), 10(2), 145163. Recuperado de www.redalyc.org/service/ redalyc/downloadPdf/1341/134144225008/6
Santos-Guerra, M. (1998). Una flecha en la diana. La evaluación como aprendizaje. Madrid: Ediciones Narcea.

Señorino, O., Patat, M., Vilanova, S. y García, M. (2014). Evaluación de las concepciones sobre el aprendizaje y la enseñanza en alumnos de nivel secundario. Revista de Evaluación Educativa, 2(2). Recuperado de http://www.redalyc. org/pdf/155/15590206.pdf

Turpo, O. (2011). Concepciones y prácticas evaluativas de los docentes del área curricular de ciencias en las instituciones de enseñanza públicas de educación secundaria. Revista Iberoamericana de Evaluación Educativa, 4(2). Recuperado de https://revistas.uam.es/index. php/riee/article/view/4465

Turpo, O. (2013). Posicionamiento de los docentes de ciencias en la evaluación de los aprendizajes: una aproximación a sus subjetividades. Educación Química, 24(2), 230-236.

Vergara, C. (2011). Concepciones de evaluación del aprendizaje de docentes destacados de educación básica. Revista Electrónica Actualidades Investigativas en Educación, 11(1), 1-30. DOI: https://doi.org/10.15517/aie. $\underline{\mathrm{v} 11 \mathrm{i} 1.10175}$

Villota, O. y Rosero, M. (2009). Las estrategias de evaluación en el proceso de enseñanza-aprendizaje. San Juan de Pasto: Editorial I. U. CESMAG. 